\title{
3D models of fractured pelvic bones for analysis of symmetry and deviation
}

Samantha Polege, Sarah McClelland, Maha Ead, David Li, Kajsa Duke, Lindsey Westover

${ }^{1}$ Department of Mechanical Engineering, University of Alberta

\begin{abstract}
Pelvic fractures are a fairly common result of falls, automobile collisions, or other direct impact incidents. Most fractures are relatively mild and not life-threatening, but severe damage may have debilitating effects on the victim's health and quality of life. Pelvic breakage often requires surgery to alleviate, but procedures may be difficult given the complex anatomical structure of the area and the specific location of the fractures. This project aimed to make the planning stage easier for surgeons by analyzing left-right symmetry of the pelvis and using this property to create, in cases where one side is fractured and the other remains intact, 3D models of reconstructed pelvic fractures. Pelvic bone has been proven to be highly symmetrical. This method would provide surgeons with a clearer idea of how to best reassemble and otherwise correct fractures in the pelvis. First, various one-sided pelvic fractures from a batch of anonymized subjects obtained from the University of Alberta Hospital were digitized in medical imaging software. Data from six subjects in total was used in the collection of results. The digitized pelvises were then used to construct 3D models. The fractured pieces were aligned in best-fit with a mirrored intact side. Colour deviation maps, which differentiated between areas of high and low symmetry by measuring the degree of point deviance against a scale, were then generated. Deviance was generally more prevalent along the fracture lines. The predefined threshold considered a maximum of $2 \mathrm{~mm}$ of deviation as highly symmetrical. The average RMS value was found to be $1.65 \mathrm{~mm}$ and the average percentage of points within 2 $\mathrm{mm}$ of difference was $85.1 \%$, indicating that the pelvic bones studied possessed a reasonably high degree of left-right symmetry. The results from this study suggest that this virtual reconstruction method is reliable for use in surgical planning for one-sided pelvic fractures. .
\end{abstract}

Key words:

Pelvic bone, pelvic, fractures, 3D model, deviation, symmetry, geomagic, CT scans, 3D modelling, bone fractures, surgery

Cite as: McClelland S., Ead M., Li D., Duke K., Westover L. 2019. 3D Models of Fractured Pelvic Bones for Analysis of Symmetry and Deviation. Alberta Academic Review, Vol 2 (2) 61-62, WISEST Special Issue (non peer-reviewed), DOI 10.29173/aar66. 
Polege et al., 2019

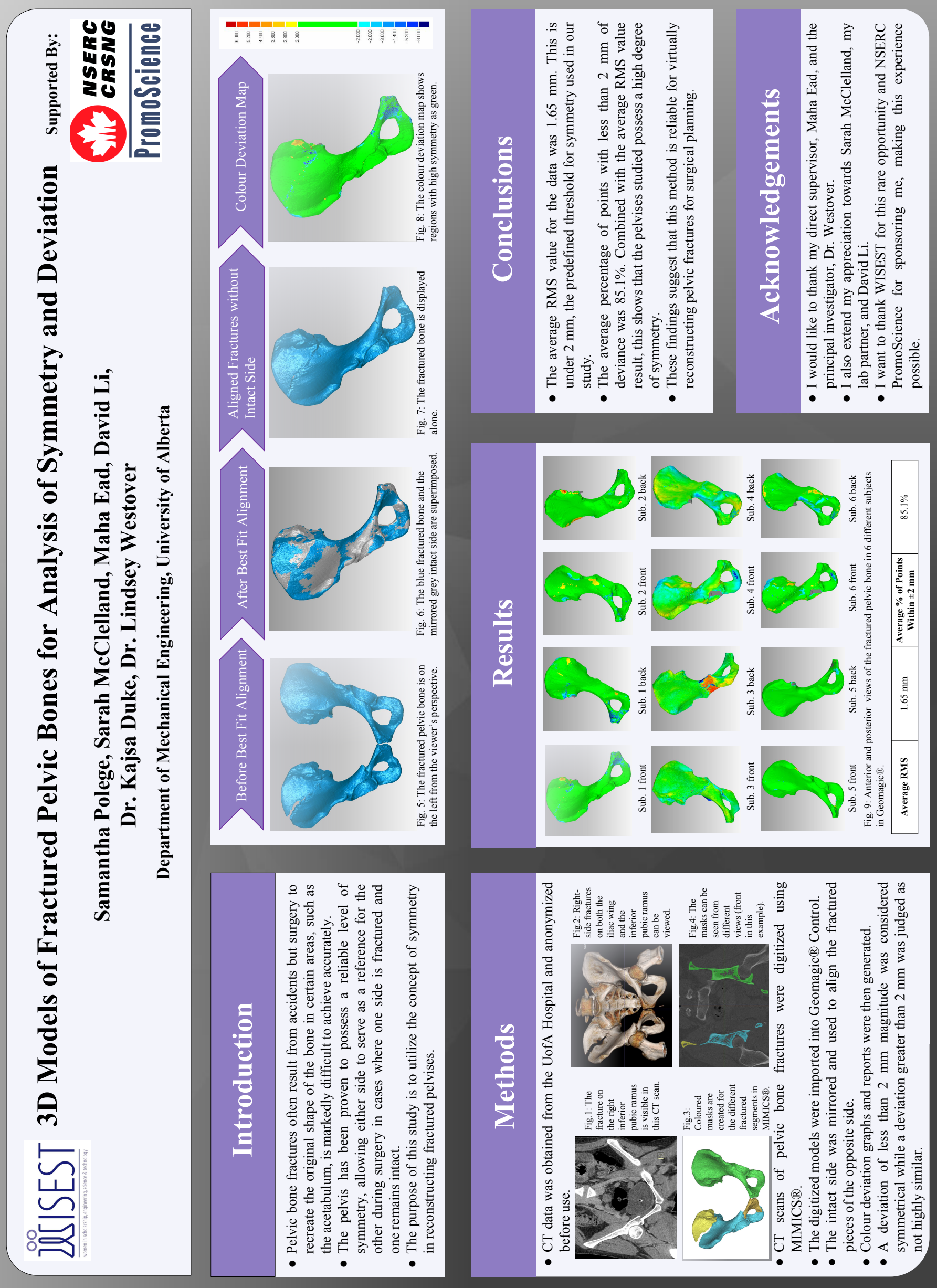

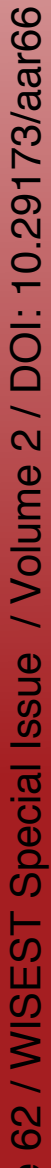

